\title{
Steroid-Prämedikation bei Brustkrebs: Adhärenz noch optimieren
}

Onkologen, Apotheker, Pflegepersonal und Patientinnen wurden über ihre Erfahrungen mit der SteroidPrämedikation vor der Anwendung von Docetaxel befragt.

D ocetaxel ruft häufig unerwünschte Reaktionen wie Überempfindlichkeitsreaktionen, Flüssigkeitsretention und Hauttoxizitäten hervor. Eine Steroid-Prämedikation kann die Inzidenz der ersten beiden genannten Nebenwirkungen deutlich reduzieren, jedoch ist die Adhärenz hier suboptimal. Für die vorliegende Untersuchung ging ein Fragebogen an Pflegepersonal, Apotheker und Onkologen, die an der Brustkrebstherapie beteiligt waren, ein zweiter an Patientinnen, die eine adjuvante Chemotherapie mit Docetaxel erhielten.

53,8 \% der Pflegenden, 19,7\% der Apotheker und 14,8\% der Onkologen beantworteten die Fragebögen (insgesamt 26,4\%). Zwei Steroid-Schemata kamen am häufigsten zur Anwendung: entweder wurden 6 Dosen (79\%) oder 2 Dosen $8 \mathrm{mg}$ Dexamethason 2-mal täglich vor der Docetaxel-Anwendung verabreicht.

Die Adhärenz gegenüber der Prämedikation sei bei den meisten Patientinnen suboptimal gewesen, so $98 \%$ der befragten Ärzte, Apotheker und Pflegenden. Die Patientinnen ließen 1-mal pro Tag (3\%), 1-mal pro Woche (33\%), 1-mal pro Monat (26\%) oder einige Male im Jahr (27\%) eine Dosis aus. Am häufigsten wurde die Dosis 24 Stunden vor der Chemotherapie weggelassen. In $65 \%$ der Institutionen gibt es lokale Protokolle zum Management von Patienten, die ihre Prämedikation nicht korrekt anwenden. Allerdings kamen in den verschiedenen Einrichtungen unterschiedliche Strategien zur Anwendung.

$82,3 \%$ der Patientinnen füllten die Fragebögen aus. $99 \%$ gaben an, dass sie ihre Prämedikation korrekt eingenommen haben. Die Patientinnen führten außerdem an, dass Steroide häufig Nebenwirkungen verursachen würden, vor allem Schlafstörungen (49\%), Hautreaktionen (22\%), Hunger und Gewichtszunahme (17\%). Darüber hinaus vermerkten $46 \%$, dass schriftliche Hinweise ihnen die regelmäßige Einnahme der $\mathrm{Me}$ dikation leichter machen könnten.

Fazit: Die Erfahrungen von medizinischem Personal zur Adhärenz bezüglich der Steroid-Prämedikation stimmen mit den Angaben der Brustkrebspatientinnen nicht überein. Ein allgemein akzeptiertes Protokoll für Prä- und Postmedikation und das Management bei falscher Anwendung sowie schriftliche Informationen könnten die Adhärenz verbessern.

Judith Neumaier

Jacobs C et al. Optimisation of steroid prophylaxis schedules in breast cancer patients receiving docetaxel chemotherapy - a survey of health care providers and patients. Support Care Cancer. 2015;23(11):3269-75.

\section{Erweiterte adjuvante Polychemotherapie beim frühen nodal positiven Mammakarzinom}

\section{Capecitabin ist aktiv beim metastasierten Mammakarzinom. In der Studie GEICAM/2003-10 wurde untersucht, ob sich das Nukleosid-Analogon bei Patientinnen mit nodal positivem Mammakarzinom in ein adjuvantes Regime auf Epirubicin- und Docetaxel-Basis integrieren lässt.}

$D_{\mathrm{E}}^{\mathrm{ie}}$ ie 15-Jahres-Überlebensdaten der Early Breast Cancer Trialists' Collaborative Group (EBCTCG) zeigten den Vorteil einer adjuvanten Polychemotherapie beim Mammakarzinom bezüglich Rezidivrisiko und Überleben. Dennoch ist die 10-Jahres-Rezidivrate mit $24 \%$ inakzeptabel hoch. Beim fortgeschrittenen Mammakarzinom ist das orale Fluoropyrimidin Capecitabin hochaktiv. In der Studie GEICAM/2003-10 wurde es einer standardmäßigen Chemotherapie auf Anthrazyklin- und Taxan-Basis zugegeben und die Wirkung auf invasives krankheitsfreies Überleben (IDFS, primärer Endpunkt), Gesamtüberleben und Sicherheit untersucht.
An der Phase-III-Studie nahmen 1.384 Patientinnen mit histologisch gesichertem HER2-negativem Mammakarzinom und axillärer Beteiligung (T1-3/ N1-3) nach Primärchirurgie teil. Sie erhielten randomisiert im Kontrollarm (EC-T) 4 Zyklen Epirubicin/Cyclophosphamid $\left(90 \mathrm{mg} / \mathrm{m}^{2} ; 600 \mathrm{mg} / \mathrm{m}^{2}\right)$ und anschließend 4 Zyklen Docetaxel (100 $\mathrm{mg} / \mathrm{m}^{2}$ ) oder im experimentellen Arm (ET-X) 4 Zyklen Epirubicin/Docetaxel $\left(90 \mathrm{mg} / \mathrm{m}^{2} ; 75 \mathrm{mg} / \mathrm{m}^{2}\right)$, gefolgt von $4 \mathrm{Zy}$ klen Capecitabin $\left(1.250 \mathrm{mg} / \mathrm{m}^{2} 2\right.$-mal täglich über 14 Tage). Nach median 6,6 Jahren waren $86 \%$ der Patientinnen im EC-T-Arm und $82 \%$ in der Gruppe mit dem Capecitabin-haltigen Regime ohne invasive Erkrankung ( $p=0,03)$. Es ergaben sich keine signifikanten Unterschiede im Gesamtüberleben. Häufigste unerwünschte Nebenwirkungen vom Grad 3/4 im EC-T- und ET-X-Arm: Neutropenie (19 vs. $10 \%)$, Fatigue (13 vs. $11 \%$ ), Diarrhö (3 vs. $11 \%$ ), Hand-Fuß-Syndrom ( 2 vs. $20 \%$ ), Mukositis (6 vs. $5 \%$ ), Erbrechen ( $5 \%$ in beiden Gruppen) und Myalgie (4,5 vs. $1 \%)$.

Fazit: Das adjuvante Standardschema EC-T führt bei Patientinnen mit einem nodal positiven Mammakarzinom im Frühstadium zu einem signifikant günstigeren invasiv krankheitsfreien Überleben als das um Capecetabin erweiterte ET-X-Regime. Es ergaben sich keine Unterschiede bzgl. des Gesamtüberlebens, wohl aber im Toxizitätsprofil.

Wolfgang Zimmermann

Martin M et al. Epirubicin plus cyclophosphamide followed by docetaxel versus epirubicin plus docetaxel followed by capecitabine as adjuvant therapy for node-positive early breast cancer: results from the GEICAM/2003-10 study. J Clin Oncol. 2015;33(32):3788-95. 